\title{
Brightening the Lives of Pregnant Women through Sunshine
}

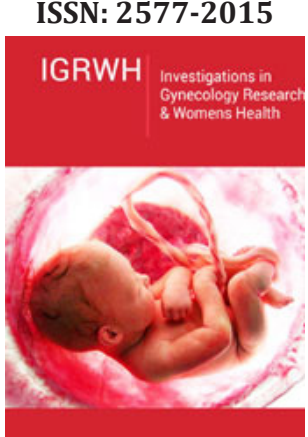

*Corresponding author: Shipra Sonkusare, Department of Obstetrics \& Gynecology, India

Submission: 傮 October 15, 2020

Published: 眥 November 20, 2020

Volume 3 - Issue 5

How to cite this article: Shwetha Guptan, Shipra Sonkusare*. Brightening the Lives of Pregnant Women through Sunshine. Invest Gynecol Res Women's Health. 3(5). IGRWH. 000573. 2020.

DOI: 10.31031/IGRWH.2020.03.000573

Copyright@ Shipra Sonkusare, This article is distributed under the terms of the Creative Commons Attribution 4.0 International License, which permits unrestricted use and redistribution provided that the original author and source are credited.

\author{
Shwetha Guptan and Shipra Sonkusare* \\ Department of Obstetrics \& Gynecology, India
}

\section{Opinion}

Appropriate antenatal care is proven to play a crucial role in ensuring good outcome of the mother and the baby. Good antenatal care ensures periodic assessment of growth and development of the baby, and supplementation of additional nutrition required for the mother. It also provides prompt recognition of any complication that befall pregnancy, one such dreaded complication being gestational diabetes mellitus. Gestational diabetes mellitus (GDM) is defined as the carbohydrate intolerance of varying severity with onset or first recognition in pregnancy. The prevalence of GDM in India is around 3.8-21\%, and it is seen more in urban women. Physical environment, ethnicity, genetics, dietary patterns, lifestyle, obesity, family history of diabetes mellitus, and history of GDM in the previous pregnancy are independent risk factors for the development of GDM.

Carbohydrate metabolism in pregnant women differs from the non-pregnant state, where there is an increased demand for metabolic fuel to compensate for fetal needs. To meet these requirements, placental hormones such as human placental lactogen, beta- hCG, and progesterone act on the body to increase peripheral resistance to insulin action, cause hypertrophy of the islets of Langerhans and hyperinsulinemia. Thus, fasting hypoglycemia and postprandial hyperglycemia are its unique features. When these changes to pathological state in pregnancy, it has various adverse effects on the mother and baby. Persistent hyperglycemia due to decreased uptake by the maternal tissues causes catecholamine release and vasoconstriction. This predisposes pregnant women to the development of hypertension, decreases placental perfusion and causes premature placental aging, intrauterine fetal growth restriction and has the potential for intrauterine fetal demise. The fetus also is at risk for birth injuries, and perinatal asphyxia, thereby increasing the perinatal morbidity and mortality. These babies should be screened for congenital heart diseases like ventricular and atrial septal defects. However, Gestational Diabetes Mellitus increases the risk of women developing Type II DM later in life.

Vitamin D, called the sunshine vitamin, is a derivative of cholesterol. The precursor for synthesis is 7-dehydrocholesterol, which under the action of ultraviolet light converts to Cholecalciferol. This molecule is further metabolized to 25-hydroxyvitamin D3 in the liver, and to $1 \alpha, 25$-dihyroxyvitamin D3 in the kidney which is the active form of vitamin D. The concentration of vitamin D is mainly dependent on environmental factors, through intake of vitamin rich diet and exposure to ultraviolet light. Its dietary sources are fish, fish liver oil, egg yolk, cheese and mushroom, out of which the richest sources are from fish. Thus, significant deficiencies are seen in strict vegetarians. It is also deficient in women with less exposure to sunlight. Vitamin D affects various critical metabolic pathways in our bodies. It promotes calcium absorption and transport into blood, from bone, intestine, and kidney. It is also responsible for regulating a part of the human genome that controls carbohydrate and lipid metabolism. 
In pregnancy, vitamin $\mathrm{D}$ plays a vital role in fetal cell differentiation and skeletal growth. Vitamin D receptors, which have been of interest in recent research, show its presence in various tissues of the body, including placenta. By its action on these receptors, vitamin D helps in immune regulation, hormone production, mitigating inflammation and maintenance of pregnancy. Vitamin D supplements are available over the counter in the form of granules and also capsules. Calcium supplements have included vitamin $\mathrm{D}$ as a part of their preparation to enhance the absorption of calcium. Many studies have hinted the positive influence of vitamin $\mathrm{D}$ on the overall health and general wellbeing of pregnant women. There is extensive research proving the benefits of vitamin D in the prevention of pregnancy associated complications like GDM, preeclampsia, and antepartum hemorrhage. This calls for a routine recommendation for supplementation of vitamin D in pregnancy. The rewards of a simple intervention of optimizing the level of vitamin $\mathrm{D}$, is yet to receive the recognition it deserves.

For possible submissions Click below: 